\title{
CUÁNDO Y CÓMO REALIZAR UNA BIOPSIA DE LAS LESIONES CUTÁNEAS FRECUENTES. LO QUE EL CIRUJANO DEBE SABER*
}

\author{
Drs. Katherine Droppelmann D. ${ }^{1}$, Karina Cataldo C. ${ }^{1}$, Montserrat Molgó N. ${ }^{1}$, \\ Ignacio Goñi E. ${ }^{2}$, Augusto León R. ${ }^{2}$, Mauricio Camus A. ${ }^{2}$, Hernán González D. ${ }^{2}$, \\ Francisco Domínguez C. ${ }^{2}$, Nicolás Droppelmann M. ${ }^{2}$, \\ 1 Departamento de Dermatología. \\ 2 Departamento de Cirugía Oncológica y Maxilofacial. División de Cirugía Facultad de Medicina Pontificia \\ Universidad Católica de Chile. \\ Santiago, Chile.
}

\section{Abstract \\ How and when to perform a skin biopsy of a pigmented lesion. What the surgeon need to know}

At present times, general surgeons are continuously faced to evaluate patients with pigmented lesions. Thus, is very important that surgeons acquire adequate knowledge not only to distinguish between suspicious lesion and non suspicious lesion, but also to correctly assess when and how to perform a skin biopsy. The early detection of melanoma and non melanoma skin cancer is one of the most important factors to achieve a better prognosis. The main objective of this article is to provide surgeons some tips and pitfalls to help accurate the evaluation and diagnosis of pigmented lesions. The authors also want to stress out the importance of the team work between surgeons and dermatologist, due that is well documented that multidisciplinary approach to skin cancer raises the possibilities of early diagnosis, adequate treatment and better results for patients with skin cancer.

Key words: Skin cancer, suspicious lesion, diagnosis, biopsy.

\section{Resumen}

En la actualidad, el cirujano continuamente se ve enfrentado a evaluar distintos tipos de lesiones cutáneas en los pacientes, por lo que debe tener conocimientos de las características que hacen que una lesión sea sospechosa o no, para evaluar correctamente cuándo y cómo realizar una biopsia de una lesión cutánea. El diagnóstico precoz, tanto del melanoma como del cáncer de piel no melanoma, ha demostrado ser clave para mejorar el pronóstico de nuestros pacientes. Este artículo pretende entregar algunas claves para afinar

*Recibido el 13 de octubre de 2014 y aceptado para publicación el 23 de marzo de 2015.

Los autores no refieren conflictos de interés.

Correspondencia: Dr. Nicolás Droppelmann ndroppel@med.puc.cl 
la evaluación y diagnóstico de las lesiones pigmentadas. Es muy importante también, recalcar la importancia del trabajo conjunto de los cirujanos con los dermatólogos, ya que la evaluación y manejo multidisciplinario mejora sustancialmente el diagnóstico, tratamiento y resultados de los pacientes con cáncer de piel.

Palabras clave: Cáncer de piel, lesiones cutáneas sospechosas, diagnóstico, biopsia.

\section{Introducción}

El cáncer de piel corresponde al cáncer más prevalente en humanos, dando cuanta de la mitad de todas las neoplasias malignas ${ }^{1-3}$. La sociedad americana del cáncer reportó 12.190 muertes por cáncer de piel en el año $2012^{3}$. El cáncer de piel no melanoma (CPNM) es más frecuente que el melanoma maligno; de este grupo, los tipos más comunes son el carcinoma basocelular (CBC) en primer lugar y el carcinoma espinocelular (CEC) en segundo lugar $^{1}$. En Estados Unidos, se diagnostican más de un millón de CPNM cada año ${ }^{1}$. La incidencia de CPNM ha aumentado anualmente entre un 3\% y $8 \%$ en todo el mundo, y se estima que aumentará en un $50 \%$ de aquí al año $2030^{2,3}$.

El CBC corresponde al $80 \%$ a $90 \%$ de todos los $\mathrm{CPNM}^{1,3}$. A pesar de no existir un registro preciso en ningún país, se estima que el CBC estaría aumentando en incidencia y prevalencia en el mundo, en relación a un mayor diagnóstico y a una mayor esperanza de vida en la población general ${ }^{1}$. Se estima que el riesgo de los caucásicos de desarrollar un $\mathrm{CBC}$ en la vida es de un $30 \%{ }^{4}$. En general el CBC no produce metástasis a distancia (riesgo menor a $0,5 \%$ ), pero su crecimiento local puede ser muy destructivo ${ }^{3}$.

El CEC corresponde aproximadamente al 16$20 \%$ de los CPNM, con una incidencia estimada de 70.000 nuevos casos anuales en Estados Unidos ${ }^{3,5}$. Se estima un aumento en la incidencia de $3-10 \%$ anual $^{5}$. El riesgo actual de un caucásico de desarrollar un CEC en la vida es de un $15 \%{ }^{5}$. Los CEC pueden producir destrucción local intensa, y existe un riesgo estimado de metástasis, vía linfática o hematógena, de 3 a $10 \%{ }^{5}$.

El melanoma representa sólo el $4 \%$ de los cánceres de piel, pero da cuenta del $65 \%$ de todas las muertes por cáncer de piel $^{3}$. Es el segundo cáncer en frecuencia en individuos entre 15 y 29 años, dando cuenta del $11 \%$ de todas las neoplasias malignas en este grupo ${ }^{6,7}$. Su incidencia ha aumentado en forma exponencial en los últimos años, se cree que principalmente por intensa exposición solar en individuos de raza blanca ${ }^{7,8}$. El riesgo actual de tener un melanoma en la vida es de 1/55 personas.

\section{Factores de riesgo}

Los factores de riesgo para desarrollar un $\mathrm{CBC}$ son pelo rubio o rojizo, ojos azules o verdes, piel clara, exposición a radiación ultravioleta (RUV) intensa intermitente o recreacional, terapia con luz ultravioleta A y psoraleno (PUVA terapia), uso de solarium, exposición a arsénico y alquitrán, exposición a radiaciones ionizantes, genodermatosis (xeroderma pigmentoso, albinismo, síndrome de Bazex, síndrome de Gorlin) e inmunosupresión (pacientes trasplantados de órganos sólidos, pacientes con VIH o linfomas) $)^{1,2}$. El CBC no se caracteriza por tener una lesión precursora conocida ${ }^{1}$.

Los CEC pueden aparecer de novo o evolucionar a partir de lesiones precursoras como queratosis actínicas (tasa de transformación a CEC de $<0,1 \%$ hasta $16 \%$ por año $)^{2,9}$. El principal factor de riesgo de CEC es la exposición solar continua u ocupacional $^{6}$. Otros factores de riesgo son: fototipo I y II según Fitzpatrick, edad mayor de 40-50 años, sexo masculino, genodermatosis, PUVA, radiaciones ionizantes, inmunosupresión, tabaquismo, alcoholismo crónico, infección por virus papiloma humano, VIH, cicatrices y procesos inflamatorios crónicos de la piel, arcenicismo crónico, exposición a carcinógenos industriales ${ }^{2,9}$.

Los factores de riesgo para desarrollar un melanoma son numerosos, siendo los más importantes historia personal o familiar de melanoma, la presencia de nevos displásicos (especialmente si son cinco o más), piel clara (fototipo I y II según Fitzpatrick) y exposición a RUV intensa intermitente o recreacional ${ }^{6,10}$. Es interesante que la exposición a RUV continua u ocupacional muestra una asociación inversa con el desarrollo de melanoma ${ }^{11}$, excepto con el subtipo léntigo maligno ${ }^{12}$. Otros factores de riesgo son historia personal de $\mathrm{CBC}$ o CEC, presencia de más de 100 nevos, nevo congénito de más de $20 \mathrm{~cm}$, pelo y ojos claros, presencia de fotodaño (queratosis actínicas, CPNM, léntigos solares y elastosis solar), sexo masculino, edad mayor de 50 años, genodermatosis e inmunosupresión ${ }^{6}$.

\section{Características clínicas}

Clínicamente, existen varios subtipos de CBC, siendo los más importantes el nodular, superficial, morfeiforme y pigmentado ${ }^{2}$. El CBC nodular es el subtipo más común de los $\mathrm{CBC}$ (50-85\%), observándose como una pápula o nódulo eritematoso con borde perlado y solevantado, múltiples telangiectasias y en algunas ocasiones, ulceración (a medida que la lesión progresa) ${ }^{2}$ (Figuras 1 y 2). El CBC superficial 


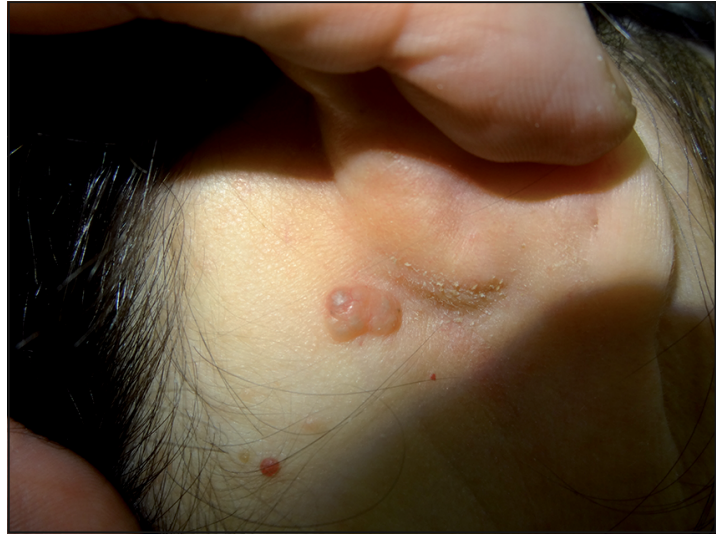

Figura 1. CBC nodular.

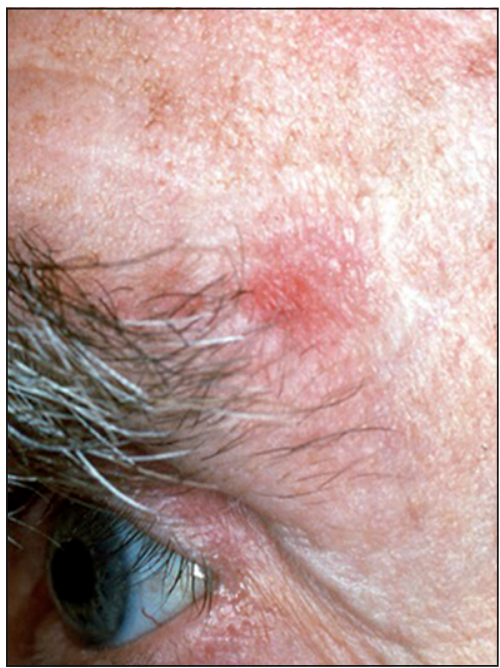

Figura 3. $\mathrm{CBC}$ morfeiforme.

se observa como una mácula o placa eritematosa con bordes levemente solevantados, puede presentar además escamas, úlceras y/o costras en su superficie ${ }^{2}$. El CBC morfeiforme se presenta como una placa, indurada, brillante, color piel-rosada o blanquecina, de bordes poco definidos y con telangiectasias desordenadas que cruzan la lesión; muchas veces simula una cicatriz (Figura 3$)^{2}$. Los CBC morfeiformes crecen extensamente antes de ulcerarse, son a menudo mal diagnosticados, su extensión es muchas veces subestimada y tienen una recurrencia mayor al $60 \%{ }^{2}$. El CBC pigmentado se puede presentar como las variantes explicadas anteriormente, pero se caracteriza por presentar pigmentación negra, café y/o azulada ${ }^{2}$.

Las queratosis actínicas, precursoras de CEC, se presentan como pápulas eritematosas, escamosas, ásperas, localizadas normalmente en superficies

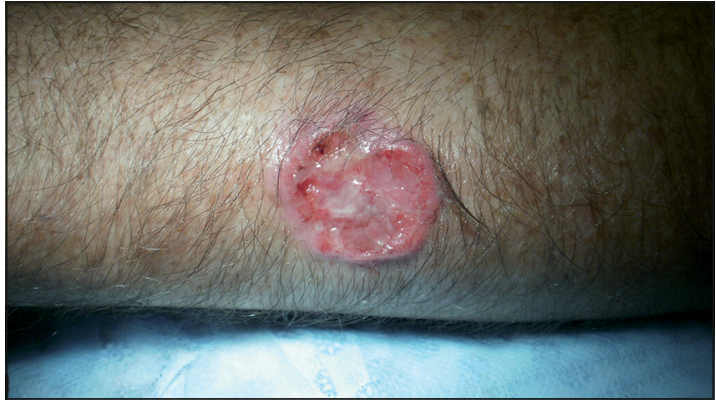

Figura 2. CBC nodular ulcerado.

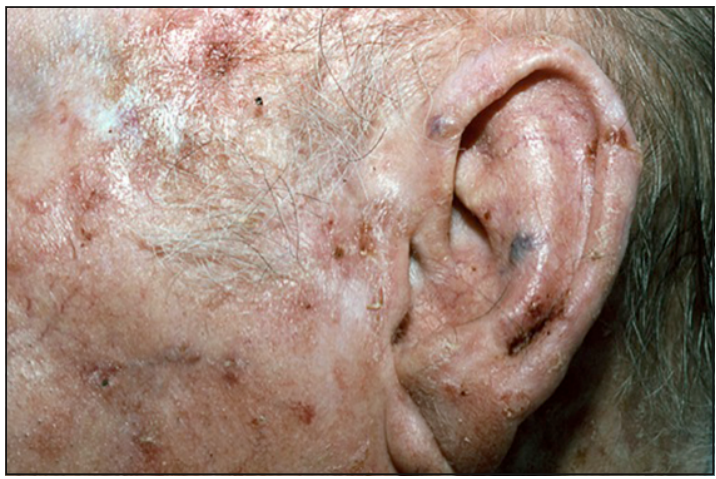

Figura 4. Queratosis actínica.

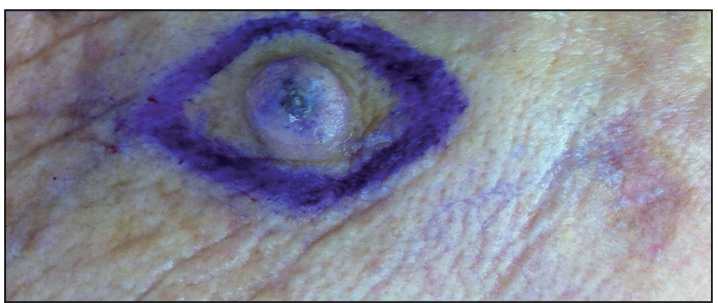

Figura 5. Queratoacantoma.

fotoexpuestas $^{2}$ (Figura 4). Cuando las queratosis actínicas se presentan engrosadas se denominan queratosis actínicas hipertróficas y tienen riesgo de haberse transformado en un CEC. Se estima que entre un 25 y $60 \%$ de los CEC se originan a partir de una queratosis actínica ${ }^{3}$. Los queratoacantomas, que corresponden a un CEC en el $20 \%$ de los casos, se presentan como tumores crateriformes, con centro ulcerado, de crecimiento rápido (4-8 semanas) $)^{13}$ (Figura 5).

Los CEC in situ (denominados enfermedad de Bowen) se presentan como pápulas o placas eritematosas, bien definidas, escamosas ${ }^{2}$ (Figura 6). Los CEC invasores se presentan como pápulas, placas o 


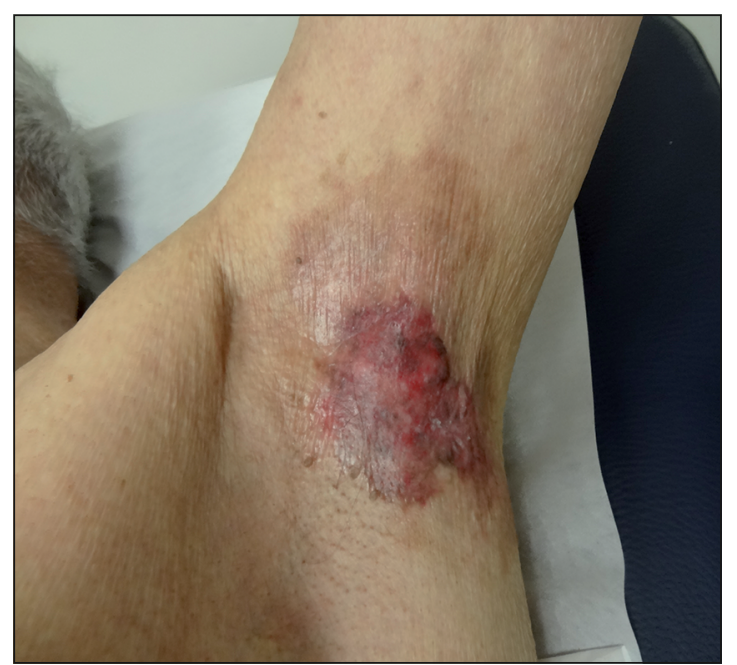

Figura 6. Enfermedad de Bowen.

nódulos, bien delimitados, de superficie escamosa o costrosa, indurados ${ }^{2}$ (Figura 7). La presencia de ulceración o costra son indicadores de invasión dérmica ${ }^{2}$.

El melanoma se caracteriza por presentarse como mácula, pápula, placa o nódulo pigmentado, heterocrómico, de bordes irregulares. Un 95\% de los melanomas tienen la historia de cambios previos ${ }^{14}$. Para guiarse clínicamente se puede usar la regla del A, B, C, D, E (A: asimetría de ejes, B: borde irregular, C: color no homogéneo, $\mathrm{D}$ : diámetro superior a $6 \mathrm{~mm}$, E: evolución) $)^{14}$; el término "patito feo": los nevos en un mismo paciente tienden a ser similares, la lesión que se desvía de este patrón individual debe ser considerada sospechosa de melanoma ${ }^{15}$, la identificación de este signo tiene un buen acuerdo interobservador, sensibilidad de $85 \%$, especificidad de $83 \%$ y precisión diagnóstica de $87 \%$ para detectar melanoma maligno, pero es altamente dependiente de la experiencia del observador y puede ser equívoco en pacientes con muchos nevos atípicos ${ }^{16} ;$ y la dermatoscopia en manos de especialistas.

Los melanomas se clasifican en 4 subtipos clínico-histológicos clásicos: de extensión superficial (70\%) (muy similar a un nevo atípico) (Figura 8), nodular (15\%) (nódulos o pápulas polipoideas, de coloración azul oscura a marrón-rojiza, el $5 \%$ de los melanomas nodulares son amelanóticos) (Figura 9), léntigo maligno (5\%) (similar a un léntigo solar, en áreas fotoexpuestas) (Figura 10), acral (5-10\%) (localizados en palmas, plantas, subungueales) $^{17}$ (Figura 11). En los casos avanzados también pueden presentar ulceración, sangrado.

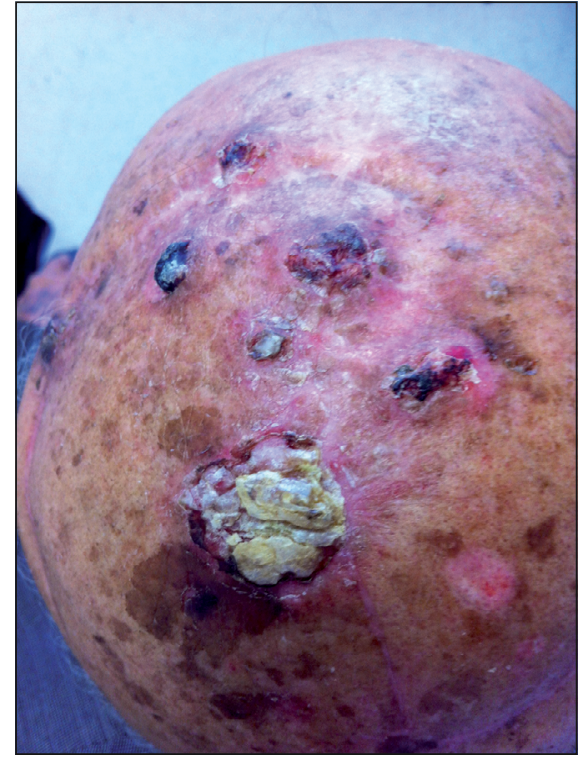

Figura 7. CEC.



Figura 8. Melanoma de extensión superficial.

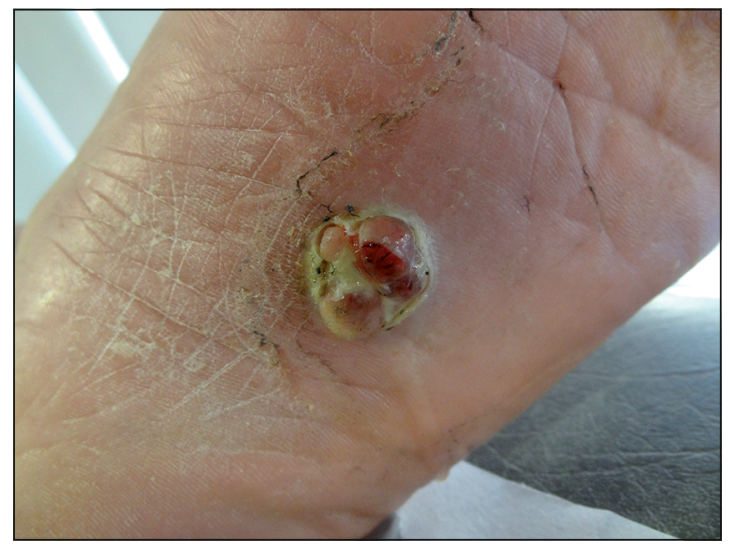

Figura 9. Melanoma nodular (amelanótico). 


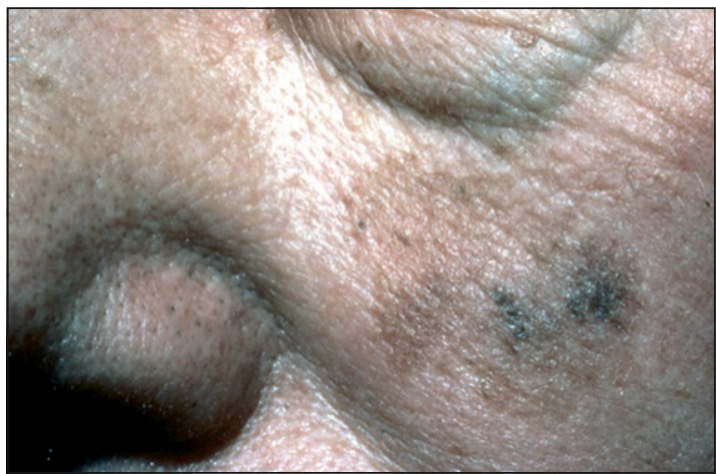

Figura 10. Léntigo maligno.

El nevo displásico o atípico se define como un nevo de al menos $5 \mathrm{~mm}$ de diámetro con un componente plano y al menos dos de las siguientes características: pigmentación variable, forma irregular y asimétrica y bordes mal definidos ${ }^{18}$ (Figura 12). Son nevos benignos, pero con desorden arquitectural $y$ atipia citológica en la histopatología ${ }^{18}$. Suelen aparecer antes de los 30 años de edad, regresar o permanecer estables a lo largo de la vida y estar relacionados con historia de quemaduras en la infancia 0 adolescencia ${ }^{18}$. La sensibilidad y especificidad del diagnóstico clínico de nevo atípico son $58,4 \%$ y $66,6 \%$ respectivamente, por lo que la mayoría de las veces queda una duda diagnóstica y es necesario extirpar la lesión para determinar si es benigna o maligna ${ }^{19}$. Los nevos atípicos son marcadores de riesgo de desarrollar melanoma, pero no necesariamente son precursores; sólo 1 en 10.000 nevos displásicos se transforman en melanoma maligno en un año ${ }^{19}$.

\section{Indicaciones de biopsia excisional}

- Nevos atípicos/sospecha de melanoma

- Queratosis actínica hipertrófica (queratosis actínica gruesa)

- Queratoacantoma

\section{Indicaciones de biopsia incisional}

- Sospecha de CBC

- Sospecha de CEC

- Nevo atípico/sospecha de melanoma muy extenso o en un área estética muy importante

\section{Técnica quirúrgica de biopsia excisional e incisional}

Los nevos atípicos deben ser extirpados idealmente en forma excisional con un margen visible libre entre 1-3 $\mathrm{mm}^{20}$. Dependiendo del tamaño, la extirpación puede realizarse con losanjo o punch, siempre alcanzando el tejido subcutáneo ${ }^{20}$. No se



Figura 11. Melanoma acral invasor.

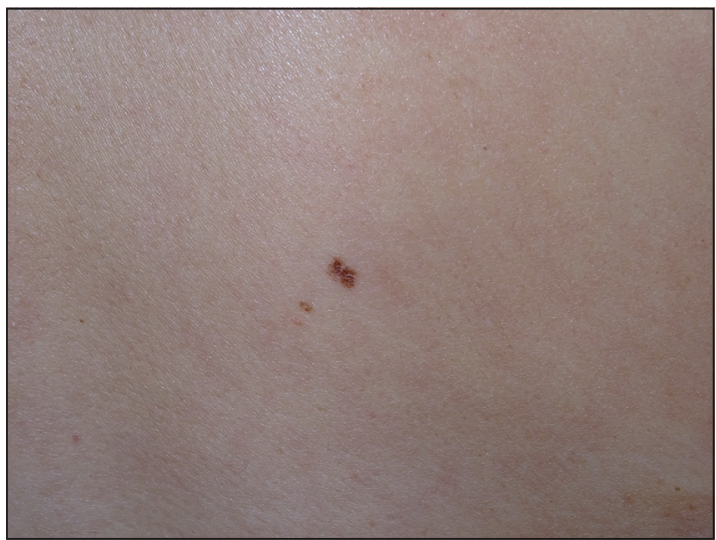

Figura 12. Nevo atípico acral.

recomiendan márgenes amplios en forma inicial, ya que las incisiones amplias pueden alterar el drenaje linfático y dificultan la extirpación posterior definitiva de márgenes en el caso de ser un melanoma por ejemplo ${ }^{20}$. Los nevos atípicos/sospecha de melanoma se deben extirpar en el sentido del drenaje linfático (eje mayor del segmento corporal ${ }^{20}$. Nunca se deben extirpar con afeitado ni electrodesecación. La biopsia incisional está indicada sólo si la lesión es muy grande o está ubicada en un lugar difícil. En estos casos, deben biopsiarse en forma incisional todas las zonas más oscuras y profundas ${ }^{20}$. Si el estudio histológico muestra un melanoma, el paciente debe ser derivado a dermatología/cirugía oncológica. El tratamiento de melanoma depende de su etapifi- 
cación, que considera profundidad de Breslow, tasa mitótica, ulceración, compromiso nodal y metástasis a distancia ${ }^{21}$. Todos los nevos extirpados deben ser enviados a estudio histológico.

Los queratoacantomas se deben extirpar en forma excisional con losanjo hasta el plano subcutáneo con un margen visible de $4 \mathrm{~mm}^{13}$.

En el caso de sospechar un CBC o CEC se debiera hacer una biopsia incisional inicial con punch/ losanjo para confirmar el diagnóstico. Si se confirma el diagnóstico los pacientes deberían ser derivados a dermatología/cirugía oncológica idealmente.

El tratamiento de elección de los CBC es el tratamiento quirúrgico ${ }^{1}$. Se ha establecido que debieran ser extirpados con un margen clínico visible de 4 $\mathrm{mm}$ y de 5 a $10 \mathrm{~mm}$ en los casos de recurrencias ${ }^{1}$. Según el tamaño y localización se puede programar un cierre primario, injerto y/o colgajo. En el caso de realizar un injerto o colgajo, se debiera tener la confirmación de los bordes libres con una biopsia rápida intraoperatoria. El objetivo es lograr bordes negativos histológicos mayores o iguales a $1 \mathrm{~mm}$. La cirugía micrográfica de Mohs se reserva para lesiones en áreas donde el resultado cosmético es importante (cabeza y cuello) y en CBC recurrentes, pues el segundo método ofrece preservación del máximo de tejido sano y la menor recurrencia a 5 años ${ }^{1,2}$. La alternativa a la cirugía en el CBC es la radioterapia, reservada para pacientes con contraindicación quirúrgica o en casos muy extensos. Otras alternativas también incluyen el curetaje (lesiones superficiales menores a $2 \mathrm{~cm}$ ), crioterapia (lesiones superficiales menores a $2 \mathrm{~cm}$ ), tratamiento tópico con Imiquimod o 5 fluor-uracilo, terapia fotodinámica, vismodegib ${ }^{1}$; ninguno de los cuales es tratamiento de primera línea.

El tratamiento de elección en el CEC también es quirúrgico ${ }^{2}$ y es de manejo exclusivo de especialistas. La cirugía micrográfica de Mohs es el tratamiento de elección en cabeza y cuello, especialmente en áreas con alto riesgo de recurrencia, como centrofacial, periorbitaria y periocular ${ }^{2}$.

\section{Conclusiones}

El cáncer de piel es el cáncer más frecuente, afecta a millones de personas en el mundo y se ha convertido en un problema de salud pública.

Conocer la clínica de las lesiones cutáneas sospechosas de cáncer de piel es imprescindible para un diagnóstico y tratamiento oportuno. Los cánceres de piel tienen riesgo de mortalidad (principalmente melanoma), riesgo de metástasis (melanoma, CEC), pueden producir destrucción local importante $(\mathrm{CBC}$, CEC).

Las lesiones cutáneas sospechosas de cáncer de piel deben ser biopsiadas en forma oportuna, ya que el diagnóstico definitivo es histopatológico.

La importancia de los nevos atípicos, además de ser marcadores de riesgo de melanoma, radica en que es muy difícil hacer el diagnóstico diferencial con un melanoma en forma clínica, ante la duda deben ser extirpados, idealmente en forma excisional.

Nunca se debe realizar una extirpación de una lesión cutánea sin ser biopsiada.

El tratamiento de elección del cáncer de piel es la cirugía ${ }^{2}$ y requiere manejo de especialistas en muchas ocasiones.

\section{Referencias}

1. Kwasniak LA, García-Zuazaga J. Basal cell carcinoma: evidence-based medicine and review of treatment modalities. Int J Dermatol. 2011;50:645-58.

2. Dubas LE, Ingraffea A. Nonmelanoma skin cancer. Facial Plast Surg Clin N Am. 2013;21:43-53.

3. Gordon R. Skin cancer: an overview of epidemiology and risk factors. Semin Oncol Nurs. 2013;3:160-9.

4. Roewert-Huber J, Lange-Asschenfeldt B, Stockfleth E, Kerl H. Epidemiology and aetiology of basal cell carcinoma. Br J Dermatol. 2007; 157:47-51.

5. Lomas A, Leonardi-Bee J, Bath-Hextall F. A systematic review of worldwide incidence of nonmelanoma skin cancer. Br J Dermatol. 2012;166:1069-80.

6. Psaty EL, Scope A, Halpern AC, Marghoob AA. Defining the patient at high risk for melanoma. Int J Dermatol. 2010;49:362-76.

7. Robinson J, Baker MK, Hillhouse J. New approaches to melanoma prevention. Dermatol Clin. 2012;30:40512.

8. Mervic L. Prognostic factors in patients with localized primary cutaneous melanoma. Acta Dermatovenerol APA 2012;21:27-31.

9. Zalaudek I, Whiteman D, Rosendahl C, Menzies SW, Green AC, Hersey P. Update on melanoma and nonmelanoma skin cáncer. Expert Rev Anticancer Ther. 2011;11:1829-32.

10. Gandini S, Sera F, Cattaruzza MS, Pasquini P, Abeni $\mathrm{D}$, Boyle P, et al. Meta-analysis of risk factors for cutaneous melanoma: I. Common and atypical naevi. Eur J Cancer 2005;41:28-44.

11. Gandini S, Sera F, Cattaruzza MS, Pasquini P, Picconi $\mathrm{O}$, Boyle P, et al. Meta-analysis of risk factors for cutaneous melanoma: II. Sun exposure. Eur J Cancer 2005;41:45-60.

12. Caini S, Gandini S, Sera F, Raimondi S, Fargnoli MC, Boniol M. Meta-analysis of risk factors for cutaneous melanoma according to anatomical site and clínicopathological variant. Eur J Cancer 2009;45:3054-63.

13. Weedon DD, Malo J, Brooks D, Williamson R. Squamous cell carcinoma arising in keratoacanthoma: a 
neglected phenomenon in the elderly. Am J Dermatopathol. 2010;32:423-6.

14. Goodson AG, Grossman D. Strategies for early melanoma detection: Approaches to the patient with nevi. J Am Acad Dermatol. 2009;60:719-35.

15. Grob JJ, Bonerandi JJ. The "ugly duckling" sign: identification of the common characteristics of nevi in an individual as a basis for melanoma screening. Arch Dermatol. 1998;134:103-4.

16. Scope A, Dusza SW, Halpern AC, Rabinovitz H, Braun RP, Zalaudek I. The "Ugly Duckling" sign. Agreement between observers. Arch Dermatol. 2008;144:58-64.

17. Scolyer RA, Long GV, Thompson JF. Evolving con- cepts in melanoma classification and their relevance to multidisciplinary melanoma patient care. Mol Oncol. 2011;5:124-36.

18. Elder DE. Dysplastic naevi: an update. Histopathology 2010;56:112-20.

19. Naeyaert JM, Brochez L. Clinical practice: dysplastic nevi. N Engl J Med. 2003;349:2233-40.

20. Tran KT, Wright NA, Cockerell CJ. Biopsy of the pigmented lesion-when and how. J Am Acad Dermatol 2008;59:852-71.

21. Balch CM, Gershenwald JE, Soong SJ, Thomson JF, Atkins MB, Byrd DR, et al. Final version of 2009 AJCC melanoma staging and classification. J Clin Oncol. 2009;27:6199-206. 